\title{
Beneficial Effects of Herbal Medicine on Susceptibility to Infection in a Patient with Immunoglobulin Deficiency
}

\author{
Zenichiro Kato ${ }^{*}$, Takahide Teramoto and Naomi Kondo
}

Department of Pediatrics, Graduate School of Medicine, Gifu University, Yanagido 1-1, Gifu 501-1194, Japan

\begin{abstract}
A case of immunoglobulin deficiency suffered from recurrent enterocolitis and otitis media was treated by Shoukenchuto, one of the Japanese herbal medicines (Kampo), and his susceptibility to infection markedly improved. The increase in the $\mathrm{T}$ cell percentage and in the lymphocyte proliferation in our patient suggests the importance of regulatory function of $\mathrm{T}$ cells for clinical improvement. The findings in our case and the previously reported cases suggest the usefulness of Japanese herbal medicine or Chinese medicine as an alternative or a supportive therapy for patients with immunological abnormalities.
\end{abstract}

Keywords: Herbal medicine, Shoukenchuto, immunoglobulin deficiency, CD4, CD8, lymphocyte proliferation.

We present a case of immunoglobulin deficiency with recurrent enterocolitis and otitis media whose clinical symptoms improved after treatment with Japanese herbal medicine (Kampo).

\section{CASE REPORT}

A 6-year-old boy developed complex partial seizures and EEG showed sporadic spikes in the central and parietal lobes. He was diagnosed as having partial epilepsy. Initially, val-

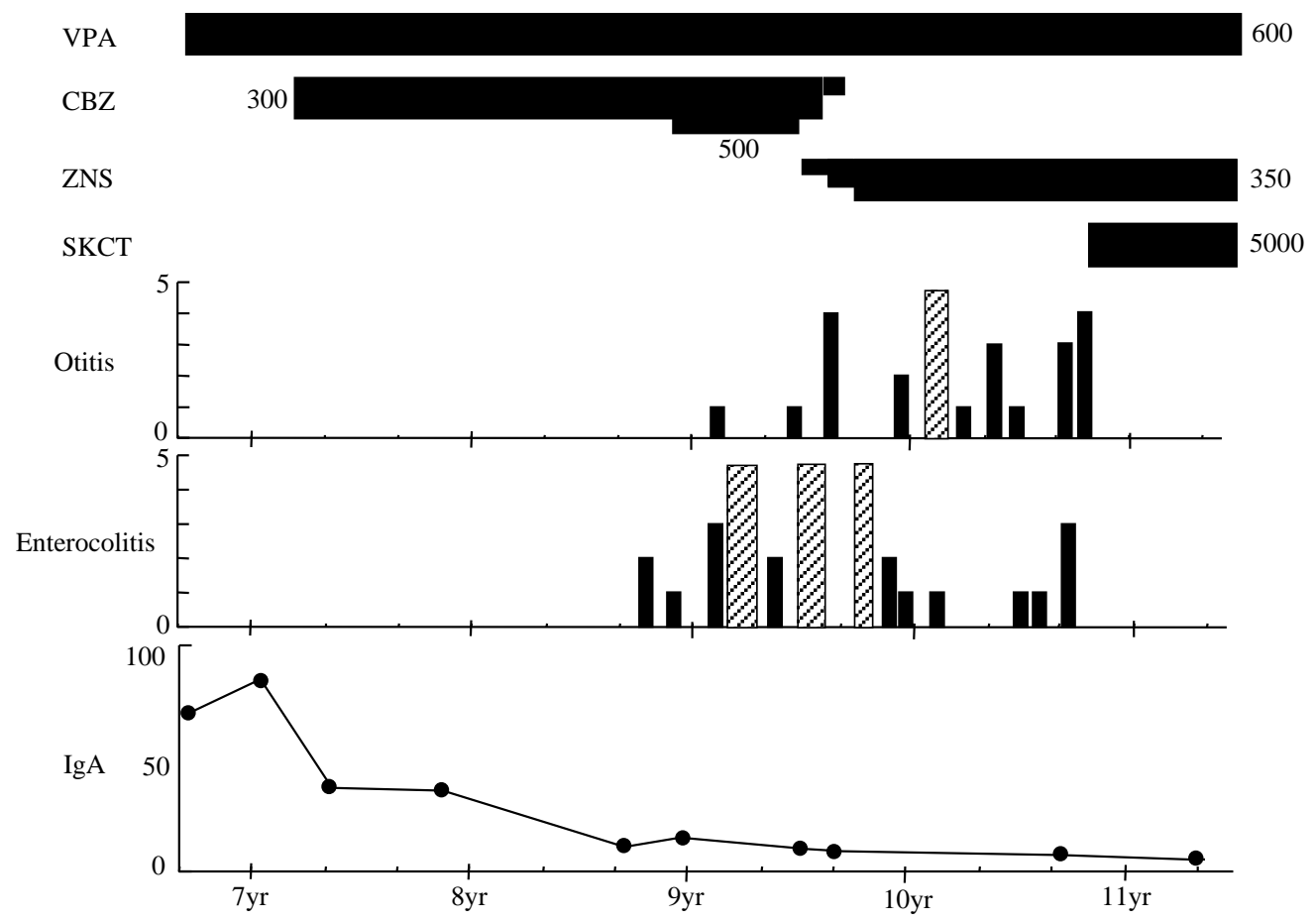

Fig. (1). Clinical changes in the patient. (Top) History of medication. VPA: valproate, CBZ: carbamazepine, ZNS: zonisamide, SKCT: Shouken-chu-to. The numbers indicate the dosages (mg/day). (Second and Third) Black bars indicate the number of hospital visits due to otitis or enterocolitis (visits/month). Shaded boxes indicate the period of admission. (Bottom) Serum IgA levels (mg/dl).

*Address correspondence to this author at the Department of Pediatrics, Graduate School of Medicine, Gifu University, Yanagido 1-1, Gifu 5011194, Japan; Tel: +81-58-230-6386; Fax: +81-58-230-6387;

E-mail: zenkato@mac.com proate was administered, but carbamazepine was required for control of his seizures. After one year of medication with carbamazepine, he frequently suffered from enterocolitis or otitis media requiring many hospital visits almost every weeks and four times of hospital admission during the 2 years. Everytime, he required to be treated with continuous drip infusion and antibiotics repeatedly (Fig. 1). His laboratory tests showed 


\section{Table1. Immunological Evaluation of the Patient}

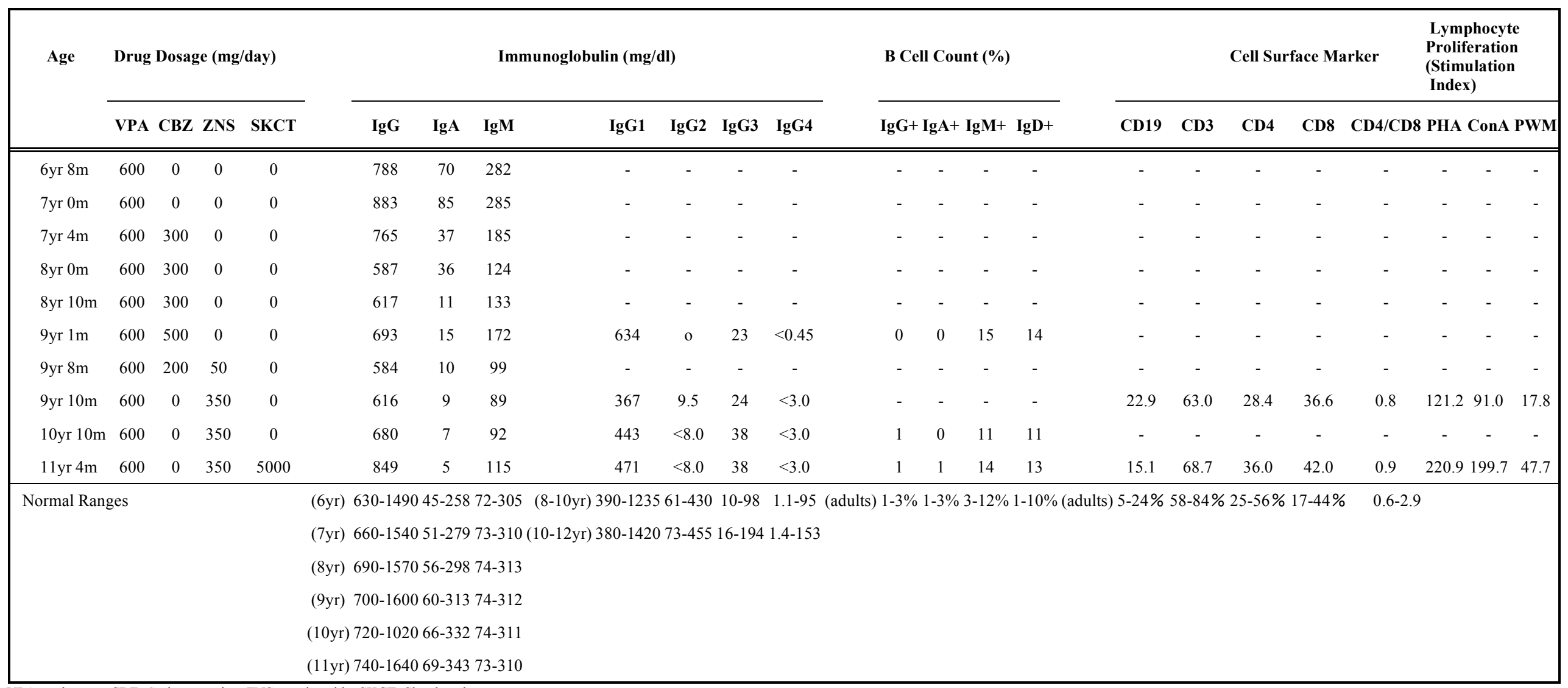


a significantly decreased IgA level and slightly decreased $\operatorname{IgG}$ level (Table 1). Further immunological examinations revealed that he was deficient in IgA, IgG2, and IgG4. The levels of IgG2 against both Hemophilus influenzae and Streptococcus pneumoniae were also markedly decreased. The levels of his serum IgA were within the normal range during valproate medication, but the levels decreased below the normal range after 7 months of carbamazepine medication (Fig. 1). He was diagnosed as having carbamazepine-related immunoglobulin deficiency as we previously described in detail [1]. Carbamazepine was replaced with zonisamide, but he still experienced recurrent enterocolitis and otitis media and his immunoglobulin levels did not normalize (Fig. 1, Table 1).

In addition to susceptibility to infections, he always complained of general fatigue and loss of appetite, although he had sufficient rest and sleep. Physical examination revealed that his lower eyelids were dark colored and his abdominal tension was weak. He was found to have general asthenia according to the conventional Chinese medicine diagnosis as compatible with the indication of Shoukenchuto (SKCT) (delicate constitution in childhood, fatigue, nervousness, and chronic gastroenteritis). We prescribed SKCT extract granules (TJ-99, TSUMURA \& Co, Japan) as $5.0 \mathrm{~g} /$ day $(0.15 \mathrm{~g} / \mathrm{kg} /$ day $)$, expecting an improvement of his general asthenia itself, not the infections. SKCT (TJ-99) contains a dried extract of the mixed crude drugs (peony root, cinnamon bark, jujube, glycyrrhiza, and ginger) and maltose.

As we expected, he no longer complained of general fatigue and loss of appetite, and the dark color of his lower eyelids almost disappeared. In addition to these improvements, surprisingly, he did not suffer from enterocolitis or otitis for 6 months after medication with SKCT, not requiring any other treatment such as antibiotics (Fig. 1). Immunological examinations after the treatment revealed that his serum $\operatorname{IgG} 2, \operatorname{IgG} 4$ and $\operatorname{Ig}$ A levels still remained very low, but the total IgG level, and the number of circulating IgA-, IgM-, and IgD-positive B cells increased. In addition, the numbers of CD3-, CD4-, and CD8-positive cells increased, while that of CD19-positive cells decreased (Table 1). Lymphocyte proliferation assays by mitogens remarkably increased compared with the values before SKCT medication.

\section{DISCUSSION}

In vitro and in vivo experiments showed that Japanese herbal medicine (Kampo) has many kinds of effects on the immune system [2], but the effect of SKCT has not been elucidated well. In a child with recurrent bronchitis, pneumonia, and enterocolitis, SKCT decreased the incidence of these diseases. Immunological examinations showed a low CD8-positive cells before treatment (CD3: 60.5\%, CD4: 47.9\%, CD8: $10.0 \%$, CD4/CD8: 4.8$)$, and the percentage normalized after the treatment (CD3: $68.1 \%$, CD4: $47.9 \%$, CD8: $19.2 \%$, CD4/CD8: 2.5) [3].

Miyakawa et al. [4] also reported the effects of Kampo medicine on a $\mathrm{T}$ cell subset in 11 children with general asthenia, which was represented by susceptibility to respiratory infection or enterocolitis. Shou-sai-ko-to $(0.2 \mathrm{~g} / \mathrm{kg})$ was administered to six children with a low percentage of CD4positive cells, while SKCT $(0.4 \mathrm{~g} / \mathrm{kg})$ was given to five patients with a low percentage of CD8-positive cells. All the patients showed clinical improvement and the percentages of their $\mathrm{T}$ cell subsets normalized. The results suggested the usefulness of $\mathrm{T}$ cell subset measurement for drug selection in addition to the conventional diagnostic classification for Chinese medicine.

Our patient was treated with SKCT for his general asthenia, not for immunological abnormalities, but his susceptibility to infection markedly improved. The increase in the $\mathrm{T}$ cell percentage, not of the B cell percentage, in our patient suggests the importance of regulatory function of $\mathrm{T}$ cells for clinical improvement. The findings in our case and the previously reported cases suggest the usefulness of Japanese herbal medicine or Chinese medicine as an alternative or a supportive therapy for patients with immunological abnormalities. However, further clinical and experimental studies should be performed to clarify the mechanisms.

\section{ACKNOWLEDGEMENT}

We thank Mr. Hideki Kashima and Mr. Masao Yamamoto for their helpful assistance.

\section{REFERENCES}

[1] Kato Z, Watanabe M, Kondo N. IgG2, IgG4, and IgA deficiency possibly associated with carbamazepine treatment. Eur J Ped 2003; 162: 209-11.

[2] Borchers AT, Sakai S, Henderson GL, et al. Shosaiko-to and other Kampo (Japanese herbal) medicines: a review of their immunomodulatory activities. J Ethnopharmacol 2000; 73: 1-13.

[3] Miyakawa S, Takamiya H, Miyakawa K, Yasuda H. Trial of Kampo medicine for primary immunodeficiency. J Trad ShinoJapanese Med 1990; 11: 256-58.

[4] Miyakawa S, Watanabe S, Itoh K. Traditional chinese medicine selection against weak children by T cell subsets. J Trad Med 1997; 14: 386-87. 